\title{
Factores asociados a sepsis abdominal en pacientes con laparotomía por trauma abdominal penetrante
}

\author{
Factors associated with abdominal sepsis in patients with laparotomy \\ due to penetrating abdominal trauma
}

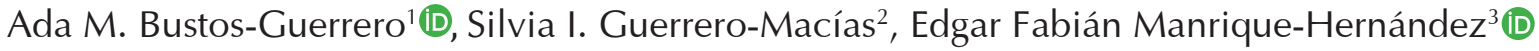 \\ 1 Médica, residente de Cirugía general, Universidad Industrial de Santander, Hospital Universitario de Santander, Bucaramanga, \\ Colombia. \\ 2 Médica, especialista en Cirugía general; profesora, Departamento de Cirugía Universidad Industrial de Santander, Bucaramanga, \\ Colombia. \\ 3 Médico, Departamento de Epidemiología, Universidad Industrial de Santander, Bucaramanga, Colombia.
}

\section{Resumen}

Introducción. Los pacientes que sufren algún tipo de trauma tienen una presentación clínica muy variable, por lo que se han diseñado pautas diagnósticas y terapéuticas con el fin de disminuir el número de laparotomías innecesarias. Las herramientas actuales para la predicción de infección intraabdominal, permiten intervenciones tempranas en los pacientes con alto riesgo y un mejor seguimiento clínico posoperatorio. El objetivo de este trabajo fue realizar un estudio de los factores asociados al desarrollo de las infecciones intraabdominales o sepsis abdominal posterior a laparotomía por trauma penetrante.

Métodos. Estudio descriptivo de una cohorte de pacientes atendidos por trauma abdominal penetrante en el Hospital Universitario de Santander, Bucaramanga, Colombia, entre enero de 2016 y diciembre de 2018. El análisis de datos se realizó en el software Stata ${ }^{\circledR}$, versión 14 (StataCorp. LP, College Station, TX, USA).

Resultados. Se incluyeron 174 pacientes con edad media de 32 años, el 10,9 \% (n=19) de los pacientes presentaron sepsis abdominal, de este grupo el 94,7\% $(n=18)$ requirieron reintervención quirúrgica $(\mathrm{p}<0,0001)$. La mortalidad general del grupo fue de 5,1\% (n=9), sin diferencia significativa entre los pacientes con o sin sepsis abdominal.

Discusión. Dentro de los diferentes desenlaces asociados a laparotomía por trauma, se describe la sepsis abdominal como una de las complicaciones que genera morbilidad significativa, con aumento en la estancia hospitalaria, necesidad de reintervención, aumento en los costos de atención y disminución en la calidad de vida, factores en concordancia con los hallazgos del presente estudio.

Palabras clave: trauma; laparotomía; control de daños; abdomen abierto; sepsis; reintervención; mortalidad.

Fecha de recibido: 15/09/2020 - Fecha de aceptación: 25/01/2021 - Fecha de publicación en línea: 07/04/2021 Correspondencia: Ada Mercedes Bustos-Guerrero, Calle 30 \# 28 - 42 Apartamento 905, Bucaramanga, Colombia. Teléfono: 3017596026. Correo electrónico: adabustosmd@gmail.com

Citar como: Bustos-Guerrero AM, Guerrero-Macías SI, Manrique-Hernández EF. Factores asociados a sepsis abdominal en pacientes con laparotomía por trauma abdominal penetrante. Rev Colomb Cir. 2021;36:493-8. https://doi.org/10.30944/20117582.842

Este es un artículo de acceso abierto bajo una Licencia Creative Commons - BY-NC-ND https://creativecommons.org/licenses/by-ncnd/4.0/deed.es 


\begin{abstract}
Introduction. Patients who suffer some type of trauma have a highly variable presentation, which is why diagnostic and therapeutic guidelines have been designed in order to reduce the number of unnecessary laparotomies. Current tools for the prediction of intra-abdominal infections allow early interventions in high-risk patients and a better postoperative clinical follow-up. The objective of this article was to study the factors associated with the development of intra-abdominal infections or abdominal sepsis after laparotomy due to penetrating trauma.
\end{abstract}

Methods. Descriptive study of a cohort of patients treated for penetrating abdominal trauma at the Santander University Hospital, Bucaramanga, Colombia, between January 2016 and December 2018. Data analysis was performed using Stata ${ }^{\circledR}$ software, version 14 (Stata corp. LP, College Station, TX, USA).

Results. A total of 174 patients with a mean age of 32 years were included, $10.9 \%(n=19)$ of the patients presented abdominal sepsis, of this group $94.7 \%(n=18)$ had a surgical reintervention $(\mathrm{p}<0.0001)$. The overall mortality of the group was $5.1 \%(n=9)$ with no significant difference between patients with or without abdominal sepsis.

Discussion. Among the different outcomes associated with laparotomy due to trauma, abdominal sepsis is described as one of the complications that generates significant morbidity, with an increase in hospital stay, the need for reoperation, an increase in care costs and a decrease in quality of life, factors in agreement with the findings of the present study.

Keywords: trauma; laparotomy; damage control; open abdomen; sepsis; reoperation; mortality.

\section{Introducción}

El trauma representa un problema de salud pública en el mundo, y corresponde a la principal causa de muerte durante las tres primeras décadas de la vida ${ }^{1}$. En Colombia, el trauma abdominal penetrante corresponde al $35 \%$ de los pacientes admitidos en centros urbanos y al $12 \%$ en centros rurales ${ }^{2,3}$.

La presentación clínica de los pacientes con trauma abdominal penetrante es variable, por lo que se han establecido pautas diagnósticas y terapéuticas con el objetivo de disminuir el número de laparotomías de precisión, que corresponden hasta del $30 \%{ }^{4-6}$ de los casos. Actualmente existe una tendencia a favor del manejo conservador, con lo cual la laparotomía exploratoria ha disminuido al $20 \%$ de los $\operatorname{casos}^{7-10}$ en pacientes con trauma abdominal. El objetivo de la laparotomía en trauma es lograr el control efectivo del sangrado, previniendo un daño fisiológico irreversible, el control de la contaminación y el reparo de otras lesiones ${ }^{1}$.

Actualmente existen herramientas para la predicción de infecciones intraabdominales, generalmente centradas en factores clínicos, co- morbilidades, tiempo operatorio, tipo de herida, número de órganos lesionados, transfusión de hemoderivados y en el índice de masa corporal ${ }^{7}$, permitiendo intervenciones tempranas en los pacientes con alto riesgo y favoreciendo el seguimiento clínico posoperatorio.

El desarrollo de la sepsis abdominal posterior a las laparotomías por trauma, se ha visto asociado a una morbilidad significativa, generando aumento de costos en la atención médica, en las reintervenciones quirúrgicas, deterioro en la calidad de vida y un aumento de 2 a11 veces en la mortalidad de estos pacientes ${ }^{8,11-12}$.

El objetivo de este trabajo fue realizar un estudio descriptivo de los factores asociados al desarrollo de infecciones intrabdominales o sepsis abdominal posterior a laparotomía por trauma penetrante, resultando en una importante herramienta para la identificación e intervención temprana en la atención de estos pacientes.

\section{Métodos}

Estudio descriptivo de una cohorte de pacientes atendidos en el Hospital Universitario de Santander, Bucaramanga, Colombia, entre enero 
de 2016 y diciembre de 2018 por trauma abdominal penetrante. Se incluyeron los pacientes que fueron llevados a laparotomía exploratoria, en ausencia de heridas en otras regiones corporales u otras intervenciones quirúrgicas. Se excluyeron los pacientes a quienes se les indicó manejo no operatorio, o fueron remitidos a otra institución.

El trauma abdominal se consideró penetrante cuando ocurrió compromiso de la fascia anterior de la pared abdominal. Se definió como laparotomía de control de daños a aquella cirugía limitada al manejo de la hemorragia y la contaminación, que requirió un segundo tiempo para el reparo definitivo de las lesiones ${ }^{9,10}$. El abdomen abierto correspondió a la estrategia de cierre temporal de la pared abdominal con el uso de dispositivos como la bolsa de poliuretano o bolsa de Bogotá. A todos los pacientes se les administró profilaxis antibiótica antes del procedimiento según el protocolo institucional.

El concepto de sepsis abdominal se realizó de acuerdo con los criterios diagnósticos, identificando principalmente la respuesta inflamatoria sistémica asociada a microorganismos aislados en cultivo de líquido o tejido abdominal, a la presencia de abscesos o colecciones encontradas en la reintervención o por imágenes diagnósticas, y al drenaje purulento a través de drenes ${ }^{13}$.

\section{Análisis estadístico}

Para la elaboración de la base de datos se utilizó el software Microsoft Excel ${ }^{\circledR}$, se calculó frecuencia y porcentaje de las variables categóricas y medidas de tendencia central, con sus valores mínimo y máximo, para el caso de las variables continuas. Se hizo análisis bivariado con las variables categóricas y los factores relacionados con sepsis abdominal. Se utilizó el método de sobrevida Kaplan Meier para analizar el tiempo de hospitalización relacionado con la presencia o ausencia de sepsis. Se realizó una regresión robusta para evaluar la asociación entre los puntos asignados a cada paciente, según el nuevo índice de severidad de la lesión (NISS, por sus siglas en inglés), el índice de trauma abdominal penetrante (PATI, por sus siglas en inglés) y la sepsis. El análisis de datos se realizó en el software Stata ${ }^{\circledR}$, versión 14 (StataCorp. LP, College Station, TX, USA).

\section{Resultados}

Se incluyeron 174 pacientes, con edad media de 32,1 años y un rango de 18 a 71 años. El 93,1 \% $(\mathrm{n}=162)$ de los participantes fueron de sexo masculino. El mecanismo de lesión fue por arma corto punzante en el 56,9 \% (n=99) y por arma de fuego en $43,1 \%(n=75)$. El 10,9\% (n=19) de los pacientes presentaron sepsis abdominal.

Se realizó tratamiento quirúrgico en un solo tiempo en el 88,5\% $(n=154)$ de los pacientes, ocurriendo sepsis en el 6,4 \% de ellos ( $n=10)$, y cirugía de control de daños en el 11,4\% ( $n=20)$, de los cuales el $45 \%(n=9)$ presentaron sepsis, (p $<0,0001$ ). Del total de pacientes que presentaron sepsis abdominal, el 94,7 \% ( $\mathrm{n}=18)$ necesitaron una reintervención quirúrgica $(\mathrm{p}<0,0001)$.

El 14,3 \% $(n=25)$ de los pacientes fueron manejados con abdomen abierto, y de ellos, el $52 \%$ $(n=13)$ cursaron con sepsis abdominal, la cual solo ocurrió en el 4,0 \% (n=6) de los pacientes a quienes se les realizó cierre temprano $(p<0,0001)$. Entre los pacientes con sepsis abdominal, durante la reintervención se dejó abdomen abierto en el $68,4 \%(n=13)$, sin embargo, en el $48 \%(n=12)$ de los pacientes con abdomen abierto, su indicación no fue sepsis abdominal.

Los pacientes con mayor puntuación en el nuevo índice de severidad de la lesión (NISS) tenían 10,7 veces más riesgo de presentar sepsis $\left(\mathrm{IC}_{95 \%}\right.$ 5,8-15,5), comparado con los que tenían una puntuación más baja, y 10,9 veces el riesgo de presentar sepsis $\left(\mathrm{IC}_{95 \%} 6,0-15,9\right)$ comparado con los que tenían una puntuación más baja en la escala PATI.

En relación al tipo de órgano lesionado, se encontró mayor frecuencia de compromiso de colon y recto, seguido de intestino delgado, órgano sólido, y páncreas-duodeno. Se identificó asociación con sepsis abdominal cuando había compromiso de estómago, órganos sólidos o páncreas-duodeno. No se encontró asociación con lesiones de intestino delgado, colon, grandes vasos o compromiso urológico (Tabla 1). 
Tabla 1. Sepsis abdominal según el órgano comprometido.

\begin{tabular}{lccc}
\hline Órgano comprometido & \% de pacientes $(\mathbf{n}=\mathbf{1 7 4})$ & Sepsis abdominal & $\mathbf{p}$ \\
\hline Estómago & $17,2 \%(\mathrm{n}=30)$ & $23,3 \%(\mathrm{n}=7)$ & $\mathrm{p}=0,017$ \\
\hline Órgano solido & $31,6 \%(\mathrm{n}=55)$ & $18,1 \%(\mathrm{n}=10)$ & $\mathrm{p}=0,037$ \\
\hline Pancreáticoduodenal & $19,5 \%(\mathrm{n}=34)$ & $20,5 \%(\mathrm{n}=7)$ & $\mathrm{p}=0,044$ \\
\hline Intestino delgado & $41,9 \%(\mathrm{n}=73)$ & $10,9 \%(\mathrm{n}=8)$ & $\mathrm{p}=0,989$ \\
\hline Colon y recto & $36,7 \%(\mathrm{n}=64)$ & $10,9 \%(\mathrm{n}=7)$ & $\mathrm{p}=0,995$ \\
\hline Grandes vasos & $17,2 \%(\mathrm{n}=30)$ & $10 \%(\mathrm{n}=3)$ & $\mathrm{p}=0,859$ \\
\hline Urológico & $3,4 \%(\mathrm{n}=6)$ & $0 \%$ & \\
\hline
\end{tabular}

Mediante el análisis de Kaplan Meier, se estableció la relación entre sepsis abdominal y estancia hospitalaria, encontrando que el 94,7 \% $(n=18)$ de los pacientes con sepsis abdominal continuaban hospitalizados después de la primera semana, y solo el $20 \%(n=31)$ de los pacientes sin sepsis habían sido dados de alta. Al día 34 de hospitalización, todos los pacientes sin sepsis habían sido dados de alta, pero solo hasta el día 104 el último paciente con sepsis tuvo egreso hospitalario (Figura 1). La mortalidad general del grupo fue de 5,1\% ( $n=9)$, sin diferencia estadísticamente significativa entre los pacientes con o sin sepsis abdominal.

\section{Discusión}

La infección del sitio operatorio de órgano o espacio se presenta aproximadamente en uno de cada cinco pacientes llevados a laparotomía por trauma (que corresponde a una incidencia del $20 \%$ ), y se ha reportado hasta un $10,6 \%$ de sepsis abdominal en este grupo de pacientes ${ }^{11,12}$. El compromiso anatómico por trauma abdominal penetrante, medido por los grados de lesión y los índices de severidad, se ha visto relacionado con los diferentes desenlaces y mortalidad en estos pacientes ${ }^{14}$.

De acuerdo al índice de severidad de la lesión (ISS, por sus siglas en inglés) y al PATI, se ha demostrado que un puntaje mayor de 15 y de 25 respectivamente, se asocia con un mayor riesgo de complicaciones sépticas de origen abdominal ${ }^{12,15,16}$, siendo más frecuente cuando existe compromiso de colon $(27,6 \%)$; intestino delgado $(20,9 \%)$ y estómago $(7,5 \%)$, atribuido generalmente a la dehiscencia anastomótica. Otros factores relacionados con el de-

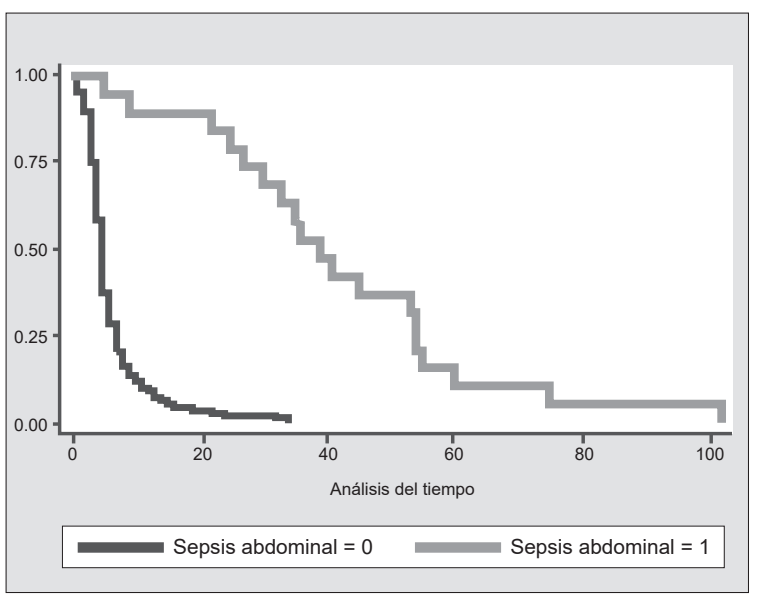

Figura 1. Gráfico de Kaplan-Meier. Análisis del tiempo de hospitalización con presencia o ausencia de sepsis abdominal.

sarrollo de sepsis abdominal son la cirugía de control de daños y el abdomen abierto ${ }^{12,17}$.

El manejo del trauma abdominal penetrante ha sufrido varios cambios en las últimas décadas, y cada vez es menos frecuente el manejo quirúrgico ${ }^{18-20}$, disminuyendo así el número de laparotomías no terapéuticas y la morbilidad asociada a una intervención quirúrgica innecesaria. En la actualidad, solo el $20 \%$ de los pacientes con trauma abdominal penetrante requieren laparotomía para el reparo de lesiones intrabdominales ${ }^{4,18-20}$.

Dentro de los diferentes desenlaces asociados a laparotomía por trauma, se describe la sepsis abdominal como una de las complicaciones que genera morbilidad significativa, con aumento en la estancia hospitalaria, necesidad de reintervención, aumento en los costos de atención y disminución en la calidad de vida ${ }^{12}$. Diferentes factores asociados 
al desarrollo de sepsis abdominal se han estudiado, estableciendo índices con variables en relación a las características de los pacientes, severidad de las lesiones y tipo de procedimiento ${ }^{7,8,11,12}$.

Los resultados obtenidos en este estudio muestran como el grado de severidad de las lesiones tiene una relación directamente proporcional con el desarrollo de la sepsis abdominal. Los pacientes con mayor puntuación de NISS y PATI tienen, respectivamente, 10,7 y 10,9 veces más riesgo de presentar sepsis abdominal comparados con los que tienen una puntuación menor a 15. Al revisar el tipo de órgano comprometido, no se encontró una correlación con lo descrito en la literatura, donde se atribuye un mayor riesgo a las lesiones de colon para el desarrollo de sepsis abdominal. En este trabajo se encontró una mayor relación con las heridas gástricas, de órgano sólido o del componente pancreáticoduodenal.

La mínima relación entre sepsis abdominal y heridas en colon encontrada en este estudio, puede explicarse por la alta tasa de lesiones de colon manejadas con colostomía. En nuestros pacientes el riesgo de sepsis abdominal se relaciona en mayor porcentaje con el grado de severidad de las lesiones, y no con el tipo de órgano comprometido.

El reparo de las lesiones se realizó en un solo tiempo quirúrgico en el 88,5\% $\%(n=154)$ de los casos, y solo en un $11,4 \%(n=20)$ se realizó laparotomía de control de daños, cifra que concuerda con la recomendación actual de disminuir este tipo de procedimientos debido a la morbilidad secundaria $\mathrm{y}$ a las complicaciones que pueden ocurrir como fístulas, sepsis abdominal y eventraciones ${ }^{10,12,16}$. Este estudio encontró un riesgo mayor de sepsis abdominal en los pacientes llevados a cirugía de control de daños (45 \%) comparado con la laparotomía en un solo tiempo quirúrgico $(6,49 \%)$, lo cual se puede explicar no solo por la severidad de las lesiones, sino además por otros factores característicos de los pacientes llevados a cirugía de control de daños como la hipotermia, acidosis y coagulopatía ${ }^{16}$.

En esta revisión, el 94,4 \% (n=18) de los pacientes con sepsis abdominal requirieron una reintervención, con hallazgos de peritonitis, colecciones intrabdominales complejas y una mayor severidad del compromiso sistémico por dehiscencia anastomótica.

El manejo de los pacientes con abdomen abierto posterior al segundo tiempo de una cirugía de control de daños o a la reintervención secundaria a cualquier causa intrabdominal, se relacionó con sepsis abdominal en el $52 \%$ de los casos, comparado con solo el $4 \%$ de los pacientes en quienes no se usó esta estrategia en su manejo. En casi la mitad de los pacientes tratados con abdomen abierto, la indicación no fue por sepsis abdominal, sino por otras causas como necesidad de nuevo empaquetamiento de lesiones hepáticas, distensión y edema de asas con dificultad para el cierre de pared y riesgo de síndrome compartimental en el segundo tiempo de una cirugía de control de daños.

\section{Conclusiones}

Los pacientes que requieren cirugía de control de daños o manejo con abdomen abierto tienen mayor riesgo de presentar sepsis abdominal. En este estudio se corroboró la relación de la sepsis abdominal con la estancia hospitalaria prolongada, lo que genera un impacto negativo en los costos de atención de los pacientes.

En la literatura mundial se informa un riesgo incrementado de mortalidad por sepsis abdominal, sin embargo, en este estudio no se encontró una diferencia significativa entre la mortalidad general y la de los pacientes con sepsis abdominal.

\section{Cumplimientos de normas éticas}

Consentimiento informado: esta investigación siguió los principios establecidos en los lineamientos de Buenas Prácticas Clínicas del Comité Internacional de Armonización y los principios éticos de la Declaración de Helsinki. Según la resolución 008430 de octubre 4 de 1993 del Ministerio de Salud de la Republica de Colombia, artículo 11, se considera como una investigación con "riesgo mínimo". Se ha preservado el anonimato y obtenido el consentimiento informado de los pacientes incluidos en el estudio. Este protocolo fue revisado y aprobado por el comité de ética institucional de la Universidad Industrial de Santander.

Conflictos de interés: los autores declaran no tener ningún conflicto de interés. 
Financiación: este trabajo fue autofinanciado.

\section{Contribución de los autores:}

Concepción y diseño del estudio: Ada M. Bustos-Guerrero, Silvia I. Guerrero-Macías.

Adquisición de datos: Ada M. Bustos-Guerrero, Silvia I. Guerrero-Macías.

Análisis e interpretación de datos: Ada M. Bustos-Guerrero, Silvia I. Guerrero-Macías, Edgar Fabián ManriqueHernández.

Redacción del manuscrito: Ada M. Bustos-Guerrero, Silvia I. Guerrero-Macías, Edgar Fabián Manrique-Hernández.

Revisión crítica: Ada M. Bustos-Guerrero, Silvia I. GuerreroMacías, Edgar Fabián Manrique- Hernández.

\section{Referencias}

1. Hirshberg A. Trauma Laparotomy: Principles and Techniques. In: Mattox KL, Moore EE, Feliciano DV, editors. Trauma. 2012. 7th ed. New York, Mc Graw Hill. p. 512-28

2. Nishimura T, Sakata H, Yamada T, Terashima M, Shirai $\mathrm{K}$, Yamada I, et al. Different patterns in abdominal stab wound in the self-inflicted and assaulted patients: an observational analysis of single center experience. Kobe J Med Sci. 2017;63:e17-21.

3. Arafat S, Alsabek MB, Ahmad M, Hamo I, Munder E. Penetrating abdominal injuries during the Syrian war: patterns and factors affecting mortality rates. Injury. 2017;48:1054-7. https://doi.org/10.1016/j.injury.2017.02.005

4. Parra-Romero G, Contreras-Cantero G, Orozco-Guibaldo D, Domínguez-Estrada A, del Campo J de JMM, Bravo-Cuéllar L. Abdominal trauma: experience of 4961 cases in western Mexico. Cir y Cir. 2019;87:183-9. https://doi.org/10.24875/CIRU.18000509

5. Pinedo-Onofre JA, Guevara-Torres L, Sánchez-Aguilar JM. Trauma abdominal penetrante TT - Penetrating abdominal trauma. Cir Ciruj. 2006;74:431-42.

6. Barbois S, Abba J, Guigard S, Quesada JL, Pirvu A, Waroquet $\mathrm{PA}$, et al. Management of penetrating abdominal and thoraco-abdominal wounds: a retrospective study of 186 patients. J Visc Surg. 2016;153:69-78. https://doi.org/10.1016/j.jviscsurg.2016.05.003

7. Walraven C Van, Musselman R. The Surgical Site Infection Risk Score ( SSIRS ): a model to predict the risk of surgical site infections. PLoS One. 2013;8:e67167. https://doi.org/10.1371/journal.pone.0067167

8. Durbin S, Deangelis R, Peschman J, Milia D, Carver T, Dodgion C. Superficial surgical infections in operative abdominal trauma patients : a trauma quality improvement database analysis. J Surg Res. 2019;243:496-502. https://doi.org/10.1016/j.jss.2019.06.101
9. Finlay IG, Edwards TJ, Lambert AW. Damage control laparotomy. Br J Surg. 2004;91:83-5. https://doi.org/10.1002/bjs.4434

10. Germanos S, Gourgiotis S, Villias C, Bertucci M, Dimopoulos N, Salemis N. Damage control surgery in the abdomen: an approach for the management of severe injured patients. Int J Surg. 2008;6:246-52.

https://doi.org/10.1016/j.ijsu.2007.05.003

11. DuBose JJ, Scalea TM, Holcomb JB, Shrestha B, Okoye $\mathrm{O}$, Inaba $\mathrm{K}$, et al. Open abdominal management after damage-control laparotomy for trauma. J Trauma Acute Care Surg. 2012;74:113-22. https://doi.org/10.1097/TA.0b013e31827891ce

12. Wei S, Green C, Kao LS, Padilla-jones BB, Truong VTT, Wade CE, et al. Accurate risk stratification for development of organ/space surgical site infections after emergent trauma laparotomy Shuyan. J Trauma Acute Care Surg. 2019;86:226-31. https://doi.org/10.1097/TA.0000000000002143

13. Garner BH, Anderson DJ. Surgical site infections an update. Infect Dis Clin NA. 2016;30:909-29. https://doi.org/10.1016/j.idc.2016.07.010

14. Guerrero SI, Serrano JP, Gelvez DJ, Delgado LJ. Índices de trauma: predicción del desenlace. Panam J Trauma, Crit Care Emerg Surg. 2015;4:172-9.

https://doi.org/10.5005/jp-journals-10030-1132

15. Gelbard RB, Hensman H, Schobel S, Khatri V, Tracy BM, Dente CJ, et al. Random forest modeling can predict infectious complications following trauma laparotomy. J Trauma Acute Care Surg. 2019;87:1125-32. https://doi.org/10.1097/TA.0000000000002486

16. Moore EE, Dunn EL, Moore JB, Thompson JS. Penetrating abdominal trauma index. J Trauma. 1981;21:439-45. https://doi.org/10.1097/00005373-198107000-00012

17. Bradley MJ, DuBose JJ, Scalea TM, Holcomb JB, Shrestha B, Okoye $\mathrm{O}$, et al. Independent predictors of enteric fistula and abdominal sepsis after damage control laparotomy: results from the prospective AAST open abdomen registry. JAMA Surg. 2013;148:947-54. https://doi.org/10.1001/jamasurg.2013.2514

18. Leenen LP. Abdominal trauma: from operative to nonoperative management. Injury. 2009;40:62-8. https://doi.org/10.1016/j.injury.2009.10.038

19. Prachalias AA, Kontis E. Isolated abdominal trauma: diagnosis and clinical management considerations. Curr Opin Crit Care. 2014;20:218-25. https://doi.org/10.1097/MCC.0000000000000074

20. Como JJ, Bokhari F, Chiu WC, Duane TM, Holevar MR, Tandoh MA, et al. Practice management guidelines for selective nonoperative management of penetrating abdominal trauma. J Trauma - Inj Infect Crit Care. 2010;68:721-33. https://doi.org/10.1097/TA.0b013e3181cf7d07 